\title{
Management of chronic subdural hematoma in Brazil
}

\author{
Manejo do hematoma subdural crônico no Brasil \\ Alisson R. Teles ${ }^{1}$, Jorge L. Kraemer ${ }^{2}$
}

Despite the burden of chronic subdural hematomas (CSDH) in neurosurgical services, the gold standard management of this condition still remains controversial. In 2012, we conducted a survey with the heads of neurosurgical services bound to the Brazilian Society of Neurosurgery (Sociedade Brasileira de Neurocirurgia) regarding the treatment strategies of CSDH $(n=49 / 54,90 \%)^{1}$. The telephone interview comprised 10 closed-ended questions about surgical approach: craniotomy vs. burr hole craniostomy vs. twist-drill craniostomy; number of burr hole craniostomies performed, if indicated (one or two); intraoperative irrigation of subdural cavity or not; use and management of drains (yes or no, duration of drainage); postoperative patient position ( flat vs. elevated head position); duration of bed rest; routine postoperative cranial image examinations; and use of corticosteroids or anticonvulsants ${ }^{1}$.

The survey demonstrated a lack of uniform approach to this common condition in Brazilian academic neurosurgical institutions. Regarding the surgical approach (Figure 1), most of the respondents preferred to use a burr hole craniostomy (85.7\%), half of them opted for one burr hole and the other half opted for two burr holes. Few centers performed the twist-drill craniostomy (6.1\%) or craniotomy $(8.2 \%)$ as the first option for treating CSDH. All neurosurgeons interviewed performed irrigation of the subdural cavity intraoperatively. Regarding postoperative management, the majority of Brazilian neurosurgical centers preferred to keep the patient's head flat after evacuation of the hematoma (59.2\%), 26.5\% preferred the elevated position and $14.3 \%$ did not recommend any specific position after surgery (i.e. the patient's preference). The duration of bed rest was no longer than two days for $75.5 \%$ of respondents. A routine postoperative computed tomography scan was performed by the majority of respondents (65.3\%). Only one center $(2 \%)$ reported the use of corticosteroids and $20.4 \%$ routinely used prophylactic anticonvulsants.

In our survey, it was observed that the majority (83.7\%) of Brazilian neurosurgical programs used a drain after

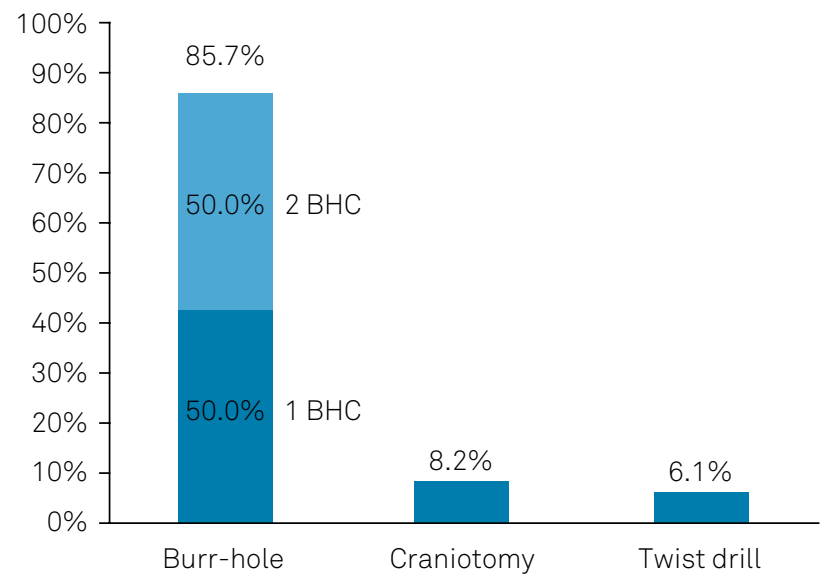

Figure 1. Results of the Brazilian national survey with the heads of academic neurosurgical institutions on the preferred surgical approach for chronic subdural hematoma.

evacuation of the CSDH (Figure 2). Most of them left the drain in the subdural $(87.8 \%)$ or subgaleal space (12.2\%). Concerning the duration of drainage, $26.8 \%$ removed the drain in 24 hours, $56.1 \%$ in 48 hours, and $17.1 \%$ usually left it for more than 72 hours.

We recently performed a broad systematic review and meta-analysis on the surgical treatment of CSDH and highlighted the best existing evidence on the subject ${ }^{2}$. Despite the lack of good quality evidence to support most of the commonly-performed neurosurgical procedures in CSDH, the use of a drain is very well established and its use is supported by a high quality randomized controlled trial ${ }^{3}$. In 2009, Santarius et al. ${ }^{3}$ published a large, high quality, and well-reported randomized controlled trial (RCT) comparing a drain versus nodrain in CSDH. The trial was stopped by the committee due to the high level of evidence of benefit from the use of the drain in lowering the recurrence rate. The long-term analysis of the RCT ( five years) showed lower mortality rates in patients who had received a subdural drain ${ }^{4}$.

${ }^{1}$ McGill University, Department of Neurology and Neurosurgery, McGill Scoliosis \& Spine Group, Montreal, Quebec, Canada;

${ }^{2}$ Hospital São José, Santa Casa de Porto Alegre, Porto Alegre RS, Brasil.

Correspondence: Alisson R. Teles; 1003, Boul. Decarie, Montreal, Canada, H4A 0A9. E-mail: alisson.r.teles@gmail.com

Conflict of interest: There is no conflict of interest to declare.

Received 20 December 2017; Received in final form 06 February 2018; Accepted 06 June 2018. 
A

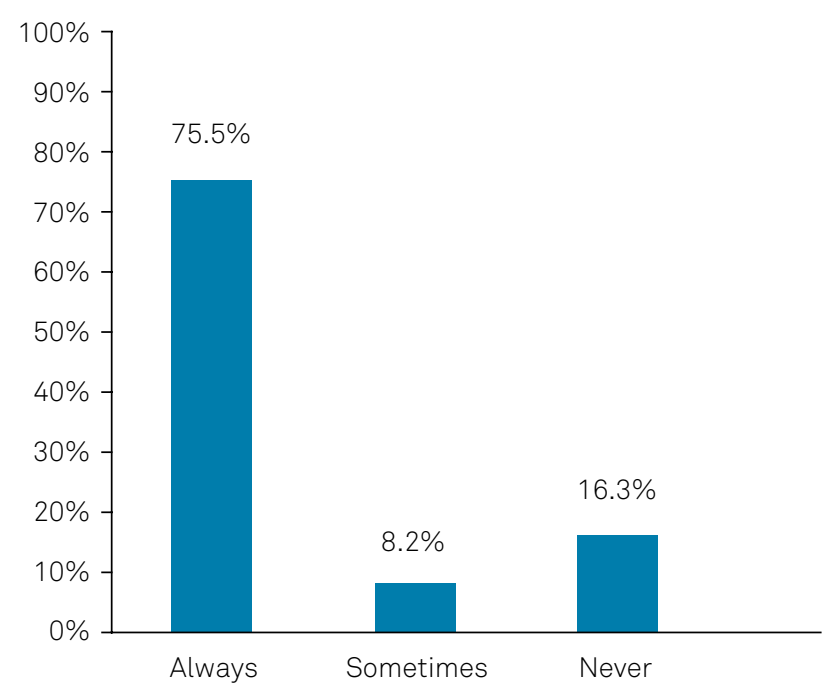

B

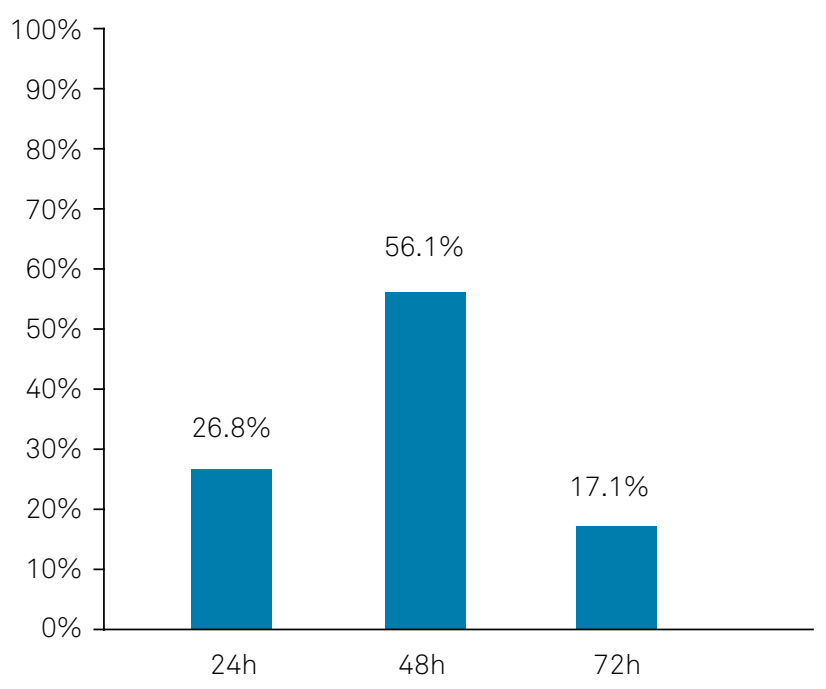

Figure 2. Results of the Brazilian national survey with the heads of academic neurosurgical institutions on (A) use of a drain in the postoperative care for chronic subdural hematomas, and (B) duration of drainage.

Surprisingly, in a recent paper published in Arquivos de Neuropsiquiatria ${ }^{5}$. Heringer et al. stated that the use of a drain does not interfere with surgical outcomes in CSDH. We recognize the importance of retrospective studies in the neurosurgical literature and congratulate the authors for their efforts. However, we respectfully disagree with their strict statement that drainage is not associated with better outcomes in CSDH. Firstly, few questions in neurosurgical practice have been answered by high quality RCTs. The benefit of drainage in CSDH is one that has ${ }^{3,4}$. Secondly, their retrospective study clearly presents several drawbacks, including (but not limited to) the unclear decision whether or not to use a drain (i.e. absence of randomization), and the obviously underpowered sample to answer this question. For example, the power analysis of the Santarius et al. ${ }^{3}$ trial estimated a sample size of 219 in each group (80\% power, alpha $=0.05)$. Thus, it is much more likely that the absence of a statistically significant association between drainage and recurrence/complications reported by Heringer et al. is due to these relevant drawbacks than a real absence of clinical benefit with drain usage, as has been already proven with high quality data ${ }^{3,4}$.

Variations in clinical practice regarding most of the procedures performed for CSDH may be caused by the absence of level I evidence for many practices ${ }^{2}$. Nevertheless, a postoperative drain is used in most Brazilian academic neurosurgical services (83.7\%). In fact, this practice is supported by high-quality neurosurgical evidence ${ }^{2,3,4}$. Another international survey ${ }^{6}$ suggested that the use of drains increased after publication of the Santarius et al. ${ }^{3}$ trial. Evidence-based national guidelines may reduce variations in practice among different centers and improve outcomes in CSDH.

\section{References}

1. Teles AR, Kraemer JK. Management of chronic subdural hematoma: a Brazilian national survey and systematic literature review. J Neurosurg. 2012;117:A424.

2. Teles AR, Falavigna A, Kraemer JK. Surgical treatment of chronic subdural hematoma: systematic review and meta-analysis of the literature. Arq Bras Neurocirur. 2016;35(2):118-27. https://doi.org/10.1055/s-0035-1571270

3. Santarius T, Kirkpatrick PJ, Ganesan D, Chia HL, Jalloh I, Smielewski $P$ et al. Use of drains versus no drains after burrhole evacuation of chronic subdural haematoma: a randomised controlled trial. Lancet. 2009 Sep;374(9695):1067-73. https://doi.org/10.1016/S0140-6736(09)61115-6
4. Guilfoyle MR, Hutchinson PJ, Santarius T. Improved long-term survival with subdural drains following evacuation of chronic subdural haematoma. Acta Neurochir (Wien). 2017 May;159(5):903-5. https://doi.org/10.1007/s00701-017-3095-2

5. Heringer LC, Sousa UO, Oliveira MF, Nunes AS, Alves KA, Zancanaro ML et al. The number of burr holes and use of a drain do not interfere with surgical results of chronic subdural hematomas. Arq Neuropsiquiatr. 2017 Nov;75(11):809-12. https://doi.org/10.1590/0004-282×20170136

6. Soleman J, Kamenova M, Lutz K, Guzman R, Fandino J, Mariani L. Drain insertion in chronic subdural hematoma: na international survey of practice. World Neurosurg. 2017 Aug;104:528-36. https://doi.org/10.1016/j.wneu.2017.04.134 